\title{
Broad-spectrum antibiotic use and poor outcomes in community-onset pneumonia: a cohort study
}

\author{
Brandon J. Webb ${ }^{1,2}$, Jeff Sorensen ${ }^{3}$, Al Jephson $^{3}$, Ian Mecham ${ }^{4}$ and \\ Nathan C. Dean ${ }^{3,5}$
}

Affiliations: ${ }^{1}$ Division of Infectious Diseases and Clinical Epidemiology, Intermountain Healthcare, Salt Lake City, UT, USA. ${ }^{2}$ Division of Infectious Diseases and Geographic Medicine, Stanford University, Palo Alto, CA, USA. ${ }^{3}$ Division of Pulmonary and Critical Care Medicine, Intermountain Healthcare, Salt Lake City, UT, USA. ${ }^{4}$ Division of Pulmonary and Critical Care, Utah Valley Regional Medical Center, Intermountain Healthcare, Provo, UT, USA. ${ }^{5}$ Division of Pulmonary Medicine, University of Utah, Salt Lake City, UT, USA.

Correspondence: Brandon J. Webb, Division of Infectious Diseases and Clinical Epidemiology, Intermountain Medical Center, 5121 South Cottonwood Drive, Murray, UT 84157, USA. E-mail: brandon.webbฉimail.org

@ERSpublications

Despite propensity-based, weighted balancing including providers and adjustment for multiple patient-level confounders, broad-spectrum antibiotics appear to be associated with increased mortality and other poor outcomes in community-onset pneumonia http://bit.ly/2DafBax

Cite this article as: Webb BJ, Sorensen J, Jephson A, et al. Broad-spectrum antibiotic use and poor outcomes in community-onset pneumonia: a cohort study. Eur Respir J 2019; 54: 1900057 [https://doi.org/ 10.1183/13993003.00057-2019].

\section{ABSTRACT}

Question: Is broad-spectrum antibiotic use associated with poor outcomes in community-onset pneumonia after adjusting for confounders?

Methods: We performed a retrospective, observational cohort study of 1995 adults with pneumonia admitted from four US hospital emergency departments. We used multivariable regressions to investigate the effect of broad-spectrum antibiotics on 30-day mortality, length of stay, cost and Clostridioides difficile infection (CDI). To address indication bias, we developed a propensity score using multilevel (individual provider) generalised linear mixed models to perform inverse-probability of treatment weighting (IPTW) to estimate the average treatment effect in the treated. We also manually reviewed a sample of mortality cases for antibiotic-associated adverse events.

Results: $39.7 \%$ of patients received broad-spectrum antibiotics, but drug-resistant pathogens were recovered in only $3 \%$. Broad-spectrum antibiotics were associated with increased mortality in both the unweighted multivariable model (OR 3.8, 95\% CI 2.5-5.9; p<0.001) and IPTW analysis (OR 4.6, 95\% CI $2.9-7.5 ; \mathrm{p}<0.001)$. Broad-spectrum antibiotic use by either analysis was also associated with longer hospital stay, greater cost and increased CDI. Healthcare-associated pneumonia was not associated with mortality independent of broad-spectrum antibiotic use. In manual review we identified antibiotic-associated events in $17.5 \%$ of mortality cases.

Conclusion: Broad-spectrum antibiotics appear to be associated with increased mortality and other poor outcomes in community-onset pneumonia. 


\section{Introduction}

Antibiotic selection is an important contributor to outcomes in community-onset pneumonia. In patients without risk factors for drug-resistant pathogens (DRPs), treatment with a narrow-spectrum $\beta$-lactam plus a macrolide is associated with decreased mortality [1]. However, it is less clear how best to identify and treat patients at high risk of DRPs. Since publication of the American Thoracic Society/Infectious Diseases Society of America healthcare-associated pneumonia (HCAP) guidelines [2] and the Surviving Sepsis Campaign guidelines [3], utilisation of broad-spectrum antibiotics such as vancomycin and piperacillintazobactam has doubled, yet the incidence of DRPs and overall outcomes remain stable [4,5]. Higher mortality has been observed in patients meeting at least one HCAP criterion [6, 7], although it remains unclear whether this effect is due to severity, comorbidities or poor functional reserve $[7,8]$. Two large propensity-adjusted studies have associated broad-spectrum antibiotic use with increased mortality in HCAP $[9,10]$. Here, we sought to clarify two important clinical questions. 1) Is broad-spectrum antibiotic use associated with poor outcomes in community-onset pneumonia after controlling for known contributors of mortality? 2) After adjusting for antibiotic selection, does HCAP remain associated with mortality risk?

\section{Methods}

\section{Design and population}

We performed a retrospective observational cohort analysis of patients $>18$ years of age admitted to the hospital with community-onset pneumonia from four emergency departments in Utah, USA from December 2011 through November 2012 [6] and from November 2014 through September 2015 [11]. Data were gathered through queries of Intermountain Healthcare's enterprise data warehouse (Salt Lake City, UT, USA). Cases were identified using International Classification of Diseases, Ninth Revision pneumonia codes and/or emergency department physician completion of an electronic pneumonia clinical decision support tool and then radiographically confirmed manually through review of emergency department chest imaging reports by study investigators. Except for Charlson Comorbidity Index (CCI), rare missing data (e.g. mental status in the emergency department not recorded during initial nurse exam) were located by manual review of provider notes. We excluded patients with more than one episode of pneumonia within 12 months and immunocompromised patients with HIV or active solid and haematological cancers. As the regression analyses adjusted for provider variability, we also excluded patients seen by providers who saw less than six patients. We also excluded patients with missing CCI data.

All antibiotics administered within the first $12 \mathrm{~h}$ after emergency department registration were included for analysis, thereby capturing empiric antibiotics administered in the emergency department as well as during the initial hospital admission. Broad-spectrum antibiotics were defined as receipt of any agent during that window with activity against either methicillin-resistant Staphylococcus aureus, such as vancomycin or linezolid, or Pseudomonas aeruginosa, such as piperacillin-tazobactam, imipenemcilastatin, meropenem, cefepime, ceftazidime or aztreonam, but excluding fluoroquinolones as they are recommended monotherapy for community-acquired pneumonia (CAP) [12]. Inadequate spectrum was defined as any initial antibiotic regimen not active against the pathogen ultimately identified.

All microbiology results were reviewed manually by the infectious disease physician investigator (B.J.W.). Positive results included organisms compatible with respiratory pathogens [13] recovered from cultures of blood, sputum, tracheal aspirate, bronchoalveolar lavage or pleural fluid, as well as urine antigens for Streptococcus pneumoniae and Legionella pneumophila and PCR assays for Mycoplasma pneumoniae and Chlamydophilia pneumoniae. DRPs were defined as organisms falling outside the spectrum of antibiotics recommended for treatment of CAP, e.g. ceftriaxone and azithromycin or respiratory fluoroquinolones, and included MRSA, P. aeruginosa, extended-spectrum $\beta$-lactamase-producing Enterobacteriaceae and other Gram-negative bacilli resistant to third-generation cephalosporins or fluoroquinolones. Clostridioides difficile infection (CDI) was defined by diagnosis $>72 \mathrm{~h}$ after admission and within 30 days of hospital discharge.

Cost (USD) was calculated as total variable costs, excluding indirect facility costs.

Approval for the study was granted by the Intermountain Healthcare institutional review board.

\section{Statistical analysis}

Simple, two-way comparisons used the Chi-squared test or Fisher's exact test for categorical variables and the unpaired t-test or Mann-Whitney U-test for continuous variables depending on distributional assumption. Hypothesis tests were two-tailed and considered statistically significant if $\mathrm{p}<0.05$.

The primary outcome was 30-day all-cause mortality and we chose the following covariate adjusters: age, sex, HCAP, CCI, electronic CURB-65 (eCURB: confusion, urea $>7 \mathrm{mmol} \cdot \mathrm{L}^{-1}$, respiratory rate $\geqslant 30$ breaths $\cdot \mathrm{min}^{-1}$, 
blood pressure $<90 \mathrm{mmHg}$ (systolic) or $\leqslant 60 \mathrm{mmHg}$ (diastolic), age $\geqslant 65$ years) [14], arterial oxygen tension $\left(\mathrm{PaO}_{2}\right)$ /inspiratory oxygen fraction $\left(\mathrm{FIO}_{2}\right)$ ratio [15], intubation status, receipt of vasopressors, number of severe CAP (sCAP) minor criteria [12], bacteraemia, length of stay, inadequate antibiotic spectrum and admitting provider. Secondary outcomes included length of stay, cost and 30-day incidence of CDI. Covariate adjusters for length of stay and cost were age, HCAP, CCI, eCURB, intubation status, receipt of vasopressors, number of sCAP criteria and provider. As we observed only 27 cases of CDI (1.4\%), we adjusted only for length of stay to avoid overfitting.

To measure the effect of broad-spectrum antibiotic use on binary outcomes (30-day mortality and CDI), we fitted unweighted multivariable regressions with logit-link functions. For $\Gamma$-distributed outcomes (length of stay and cost), we fitted unweighted multivariable regressions with log-link functions. Effect estimates are reported as exponentiated $\beta$ coefficients $\left(e^{\beta}\right)$, interpreted as odds ratios for binary outcomes and as multipliers for $\Gamma$-distributed outcomes.

As the decision to prescribe broad-spectrum antibiotics varies by an individual provider's assessment of both severity of illness and likelihood of a DRP, we recognised the possibility of indication bias in estimating the causal effect of broad-spectrum antibiotics on outcomes. To mitigate this bias, we conducted sensitivity analyses to measure the average treatment effect on the treated (ATT) by inverse-probability of treatment weighting (IPTW) [16-18]. To do this, we developed a propensity score using multilevel generalised linear mixed models with provider-level random intercepts and the following covariates: age, sex, eCURB, $\mathrm{PaO}_{2} / \mathrm{FIO}_{2}$ ratio, number of sCAP criteria, intubation status, receipt of vasopressors, CCI, HCAP, diabetes mellitus, moderate-to-severe liver disease, paraplegia and hemiplegia, congestive heart failure, and cancer. Lastly, to address the possibility that some patients may not have received care commensurate with severity due to goals of care decisions, we also conducted identical subgroup analysis in a cohort restricted only to patients admitted to the intensive care unit (ICU).

We conducted statistical model diagnostics using calibration plots and discrimination via receiver operator characteristic curves for all binary outcome models and de-trended quantile-quantile plots for continuous outcome variables [19]. Refer to the supplementary material for a detailed summary of model diagnostics. To assess the impact of IPTW on balancing variables between the broad-spectrum and non-broad-spectrum groups, we plotted the unweighted and the IPTW standardised differences and variance ratio between treatment groups for each covariate in the primary model [18]. Statistical analyses were conducted using R version 3.5.1 (R Foundation for Statistical Computing, Vienna, Austria) and SPSS version 22.1 (IBM, Armonk, NY, USA).

Finally, to investigate unmeasured variables and real-world biological plausibility of observations noted during analysis, the infectious disease physician investigator (B.J.W.) performed a standardised manual review of $20 \%$ of randomly selected mortality cases. Cases were annotated when any of the following were considered to directly contribute to demise: 1) comorbidities and in-hospital complications, including acute kidney injury, blood disorders, cardiac events, chronic pulmonary disease, falls/injury, hypersensitivity, metastatic malignancy, neurological/cognitive and thrombosis; 2) change of goals of care leading to palliative management or withdrawal of care; 3 ) advanced age (>75 years); 4) severity (referring to haemodynamic and/or respiratory compromise attributable to pneumonia); 5) adverse events attributable to broad-spectrum antibiotic and not to any other more obvious cause, including acute kidney injury, anaphylaxis, CDI, cytopenia, encephalopathy, hepatotoxicity, hypersensitivity (non-anaphylactic) and skin/mucosal reaction (e.g. Stevens-Johnson or similar disorders).

\section{Results}

We identified 2198 patients who met our eligibility criteria. We excluded 37 patients cared for by low-volume providers (see Methods) and another 166 patients with missing CCI data. The final cohort for analysis included 1995 patients.

Median age of patients was 67 years and $51.5 \%$ were female (table 1). Median CCI was 3 and mean eCURB predicted 30 -day mortality was $6.1 \%$. A bacterial pathogen was identified in $14.2 \%$ of cases; the incidence of identified DRPs was $3 \%$, but $39.7 \%$ of cases received initial empiric broad-spectrum antibiotics. Clinical and demographic data by broad-spectrum antibiotic use are shown in table 1. Patients in the broad-spectrum group more frequently met HCAP criteria (36.4\% versus 7.4\%). The broad-spectrum group also had higher eCURB predicted 30 -day mortality ( $8.1 \%$ versus $5.0 \%$ ), lower $\mathrm{PaO}_{2} / \mathrm{FIO}_{2}$ ratio (248.2 versus 269.5) and more sCAP criteria (2 versus 1 ). Intubation (13.3\% versus $2.8 \%$ ) and vasopressor use $(13 \%$ versus $1.7 \%)$ were more common in the broad-spectrum group. Bacterial pathogens were recovered more often in this group ( $21.2 \%$ versus $9.9 \%)$, as were DRPs $(7.3 \%$ versus $0.6 \%$ ). Inadequate empiric antibiotic spectrum was not significantly different in the broad-spectrum group ( $1.4 \%$ versus $0.6 \%$; $\mathrm{p}=0.1$ ). Observed 30 -day mortality was $18.3 \%$ versus $4.4 \%$. 
TABLE 1 Baseline characteristics stratified by receipt of any broad-spectrum antibiotic

\begin{tabular}{|c|c|c|}
\hline & $\begin{array}{c}\text { Any broad-spectrum } \\
\text { antibiotic }\end{array}$ & $\begin{array}{c}\text { No broad-spectrum } \\
\text { antibiotic }\end{array}$ \\
\hline Subjects & 731 & 1264 \\
\hline Age years & $67(54-79)$ & $67(52.8-79)$ \\
\hline Female & 50.5 (369) & $52.1(658)$ \\
\hline Charlson Comorbidity Index & $3(2-5)$ & $3(1-4)$ \\
\hline Diabetes mellitus & $45.3(331)$ & $37.6(475)$ \\
\hline Chronic pulmonary disease & $67.4(493)$ & $67.3(851)$ \\
\hline Congestive heart failure & 44.5 (325) & $33.1(418)$ \\
\hline Dementia & $6.2(45)$ & $5(63)$ \\
\hline Renal disease & $35(256)$ & 25.9 (327) \\
\hline eCURB $\%$ & $8.1 \pm 11.0$ & $5.0 \pm 7.1$ \\
\hline Vasopressors & 13 (95) & $1.7(22)$ \\
\hline Intubation & $13.3(97)$ & $2.8(36)$ \\
\hline sCAP minor criteria & $2(1-3)$ & $1(1-2)$ \\
\hline $\mathrm{PaO}_{2} / \mathrm{FlO}_{2}$ ratio & $248.2(188.6-303.8)$ & $269.5(220.9-319.1)$ \\
\hline Multilobar infiltrates & $51.3(375)$ & $43.7(552)$ \\
\hline Confusion & $17.6(129)$ & $5.2(66)$ \\
\hline $\mathrm{BUN}>19 \mathrm{mg} \cdot \mathrm{dL}^{-1}$ & $58.3(426)$ & 47.2 (597) \\
\hline White blood cell count $<4000 \mathrm{~mm}^{-3}$ & $4.0(29)$ & $2.4(30)$ \\
\hline Platelets $<100000 \mathrm{~mm}^{-3}$ & $6.3(46)$ & $3.5(44)$ \\
\hline Tachypnoea & $15.2(111)$ & $8.7(110)$ \\
\hline Temperature $<36^{\circ} \mathrm{C}$ & $13.5(98)$ & $9.1(115)$ \\
\hline Systolic blood pressure $<90 \mathrm{mmHg}$ & $8.9(65)$ & $2.8(36)$ \\
\hline Corticosteroids on admission & $26(189)$ & $23(292)$ \\
\hline Pleural effusion & $33.9(248)$ & 20.5 (259) \\
\hline HCAP & $36.4(266)$ & $7.4(94)$ \\
\hline Bacterial pathogen identified & $21.6(158)$ & $9.9(125)$ \\
\hline Bacteraemia & $5.9(43)$ & $2.8(35)$ \\
\hline Drug-resistant pathogen & $7.3(53)$ & $0.55(7)$ \\
\hline Methicillin-resistant Staphylococcus aureus & $4.0(29)$ & $0(0)$ \\
\hline Antibiotic-resistant Gram-negative bacilli & $3.7(27)$ & $0.55(7)$ \\
\hline Inadequate antibiotic therapy & $1.4(10)$ & $0.6(7)$ \\
\hline Length of stay $h$ & $101(64.5-188)$ & $63.5(41-92)$ \\
\hline Cost $\times 10^{3}$ USD & $12.2(6.9-22.8)$ & $6(4.2-9)$ \\
\hline Clostridioides difficile infection & $2.9(21)$ & $0.5(6)$ \\
\hline 30-day mortality & $18.3(134)$ & $4.4(55)$ \\
\hline
\end{tabular}

Data are presented as $n$, median (interquartile range), $\%(n)$ or mean \pm SD. eCURB: electronic CURB-65 (confusion, urea $>7 \mathrm{mmol} \cdot \mathrm{L}^{-1}$, respiratory rate $\geqslant 30$ breaths $\cdot \mathrm{min}^{-1}$, blood pressure $<90 \mathrm{mmHg}$ (systolic) or $\leqslant 60 \mathrm{mmHg}$ (diastolic), age $\geqslant 65$ years) predicted 30-day mortality; $\mathrm{PaO}_{2}$ : arterial oxygen tension; $\mathrm{FlO}_{2}$ : inspiratory oxygen fraction; SCAP: severe community-acquired pneumonia [12]; BUN: blood urea nitrogen; HCAP: healthcare-associated pneumonia.

\section{Unweighted multivariable regressions}

In the unweighted multivariable regressions (table 2), broad-spectrum antibiotic use was associated with increased mortality risk (OR 3.8, 95\% CI 2.5-5.9; $\mathrm{p}<0.001$ ). HCAP was not associated with mortality (OR $1.2,95 \%$ CI $0.8-1.9)$. We also observed significant increases in length of stay ( $e^{\beta} 1.7,95 \%$ CI $1.5-1.8$; $\mathrm{p}<0.001)$, cost $\left(\mathrm{e}^{\beta} 1.8,95 \%\right.$ CI 1.7-2.0; $\left.\mathrm{p}<0.001\right)$ and CDI (OR 3.9, 95\% CI 1.6-10.9; $\mathrm{p}=0.008$ ) associated with broad-spectrum antibiotic use (table 3). In the ICU subgroup, broad-spectrum antibiotic use remained associated with increased mortality risk (OR 3.4, 95\% CI 1.8-6.3; p<0.001).

\section{ATT using IPTW multivariable regressions}

In sensitivity analyses using IPTW, the ATT for broad-spectrum antibiotic use was associated with increased mortality (OR 4.6, 95\% CI 2.92-7.45; $\mathrm{p}<0.001$ ) (table 2), length of stay ( $\mathrm{e}^{\beta} 1.52,95 \%$ CI 1.4-1.6; $\mathrm{p}<0.001)$, cost $\left(\mathrm{e}^{\beta} 1.7,95 \%\right.$ CI 1.6-2.8; $\left.\mathrm{p}<0.001\right)$ and CDI (OR 5.8, 95\% CI 1.9-27.5; $\left.\mathrm{p}=0.008\right)$ (table 3). By IPTW-ATT, HCAP remained without mortality association (OR 1.3, 95\% CI 0.8-1.9). As depicted in figure 1, IPTW improved the covariate balance between treatment groups. In the IPTW-ATT analysis for the ICU subgroup, the ATT for broad-spectrum antibiotic use was associated with increased mortality (OR 4.0, 95\% CI 2.2-7.7; p<0.001). 
TABLE 2 Unweighted and inverse-probability treatment weighting (IPTW) multivariable regression effects of broad-spectrum antibiotics on 30-day mortality

\begin{tabular}{|c|c|c|c|c|}
\hline & $\begin{array}{c}\text { Primary regression } \\
\text { OR }(95 \% \mathrm{CI})\end{array}$ & p-value & $\begin{array}{l}\text { IPTW-ATT } \\
\text { OR }(95 \% \text { CI })\end{array}$ & p-value \\
\hline (Intercept) & $0.04(0-0.38)$ & 0.012 & $0.01(0-0.27)$ & 0.008 \\
\hline Broad-spectrum antibiotics & $3.82(2.48-5.92)$ & $<0.001$ & $4.61(2.92-7.46)$ & $<0.001$ \\
\hline $\mathrm{Age}^{\#}$ & $2.16(1.68-2.82)$ & $<0.001$ & $2.51(1.9-3.38)$ & $<0.001$ \\
\hline Female & $1.13(0.78-1.63)$ & 0.522 & $1.11(0.74-1.67)$ & 0.608 \\
\hline eCURB ${ }^{\#}$ & $1.15(0.97-1.36)$ & 0.107 & $1.16(0.97-1.38)$ & 0.103 \\
\hline $\mathrm{PaO}_{2} / \mathrm{F}_{1 \mathrm{O}_{2}}$ ratio $\#$ & $0.99(0.79-1.22)$ & 0.902 & $1.02(0.8-1.28)$ & 0.889 \\
\hline sCAP & $1.7(1.41-2.05)$ & $<0.001$ & $1.74(1.42-2.14)$ & $<0.001$ \\
\hline Intubation & $1.22(0.62-2.37)$ & 0.559 & $1.4(0.7-2.75)$ & 0.335 \\
\hline Vasopressors & $2.53(1.29-5.01)$ & 0.007 & $2.55(1.31-5)$ & 0.006 \\
\hline Inadequate antibiotic therapy & $5.34(1.1-23.19)$ & 0.03 & $5.56(1.11-24.92)$ & 0.029 \\
\hline Bacteraemia & $1.53(0.68-3.27)$ & 0.291 & $1.54(0.68-3.34)$ & 0.282 \\
\hline Length of stay ${ }^{\#}$ & $0.82(0.66-0.99)$ & 0.045 & $0.7(0.56-0.86)$ & 0.001 \\
\hline Charlson Comorbidity Index & $0.99(0.91-1.09)$ & 0.91 & $0.92(0.83-1.01)$ & 0.088 \\
\hline HCAP & $1.19(0.76-1.85)$ & 0.449 & $1.24(0.8-1.93)$ & 0.332 \\
\hline
\end{tabular}

ATT: average treatment effects on the treated; eCURB: electronic CURB-65 (confusion, urea $>7 \mathrm{mmol} \cdot \mathrm{L}^{-1}$, respiratory rate $\geqslant 30$ breaths. $\mathrm{min}^{-1}$, blood pressure $<90 \mathrm{mmHg}$ (systolic) or $\leqslant 60 \mathrm{mmHg}$ (diastolic), age $\geqslant 65$ years) predicted 30 -day mortality; $\mathrm{PaO}_{2}$ : arterial oxygen tension; $\mathrm{FlO}_{2}$ : inspiratory oxygen fraction; sCAP: severe community-acquired pneumonia [12]; HCAP: healthcare-associated pneumonia. \#: continuous variables results are reported as exponentiated $\beta\left(e^{\beta}\right)$ coefficients, interpreted as multipliers.

In the manual review of mortality cases $(n=40), 26(65 \%)$ received broad-spectrum antibiotics. Mortality occurred prior to hospital discharge in 21 cases (53\%). Comorbidities and complications were major contributors to mortality in 37 patients $(92.5 \%)$; of these, the most common were neurological/cognitive $(\mathrm{n}=9(22.5 \%))$, chronic pulmonary $(\mathrm{n}=9(22.5 \%))$, metastatic malignancy $(\mathrm{n}=8(20 \%))$, acute kidney injury $(n=8(20 \%))$ and cardiac events $(n=4(10 \%))$. Palliative goals of care led to withdrawal of care or discharge to hospice in 21 cases $(52.5 \%)$. Advanced age ( $>75$ years) contributed to mortality in 18 cases $(45 \%)$ and severity of illness in 18 cases (45\%). Consequences of broad-spectrum antibiotics contributed to mortality in seven cases (17.5\%); of these, acute kidney injury attributable to concomitant vancomycin and piperacillin-tazobactam was identified in three cases (7.5\%), vancomycin alone in two cases (5\%), CDI in two cases (5\%) and there was one case of cefepime-associated encephalopathy (2.5\%). One patient treated with ceftriaxone-azithromycin developed CDI associated with mortality.

\section{Discussion}

This is the first study to simultaneously evaluate the relative effects of broad-spectrum antibiotic use and HCAP status on outcomes in a community-onset pneumonia cohort that includes both CAP and HCAP patients and patient-level microbiology data. Demographics and comorbidities were generally similar between patients receiving broad-spectrum antibiotics and those who did not. Interestingly, we observed only modest differences between groups in objective severity measures such as $\mathrm{PaO}_{2} / \mathrm{FIO}_{2}$ ratio, eCURB predicted 30-day mortality and SCAP criteria, but more pronounced differences in process measures, including vasopressor use or mechanical ventilation. This supported our a priori hypothesis that both

TABLE 3 Unweighted and inverse-probability treatment weighting (IPTW) multivariable regression effects of broad-spectrum antibiotics on secondary outcomes

\begin{tabular}{lcccc} 
Outcome & $\begin{array}{c}\text { Unweighted regression } \\
\mathbf{e}^{\mathbf{\beta}}(\mathbf{9 5 \%} \mathbf{C l})^{\#}\end{array}$ & $\mathbf{p}$-value & $\begin{array}{c}\text { IPTW-ATT } \\
\mathbf{e}^{\mathbf{\beta}}(\mathbf{9 5 \%} \mathbf{C I})^{\#}\end{array}$ & $\mathbf{p}$-value \\
\hline Length of stay & $1.66(1.53-1.8)$ & $<0.001$ & $1.52(1.41-1.63)$ & $<0.001$ \\
Cost & $1.83(1.68-2.01)$ & $<0.001$ & $1.7(1.57-1.84)$ & $<0.001$ \\
Clostridioides difficile infection & $3.85(1.55-10.93)$ & 0.006 & $5.79(1.86-27.51)$ & 0.008 \\
\hline
\end{tabular}

ATT: average treatment effects on the treated. ${ }^{\#}$ : exponentiated $\beta$ coefficients $\left(e^{\beta}\right)$ are interpreted as multipliers for $\Gamma$-distributed variables (length of stay and cost) and as odds ratios for binary outcomes (C. difficile infection). 
a)

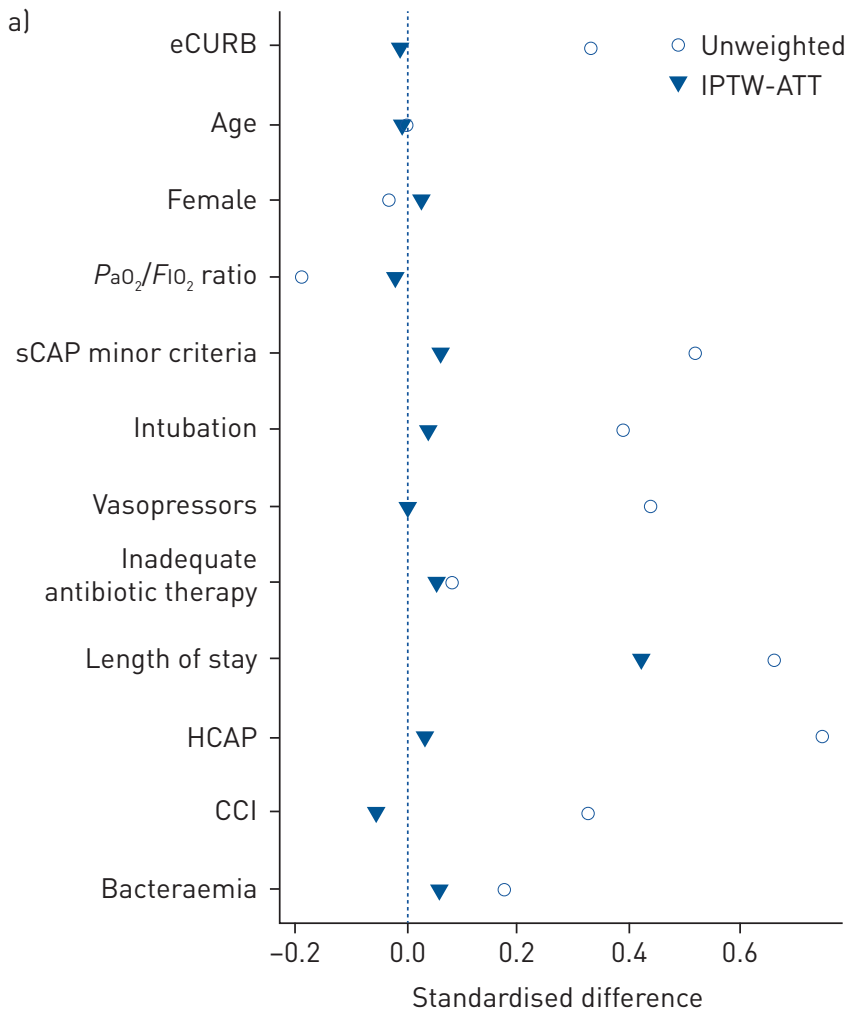

b)

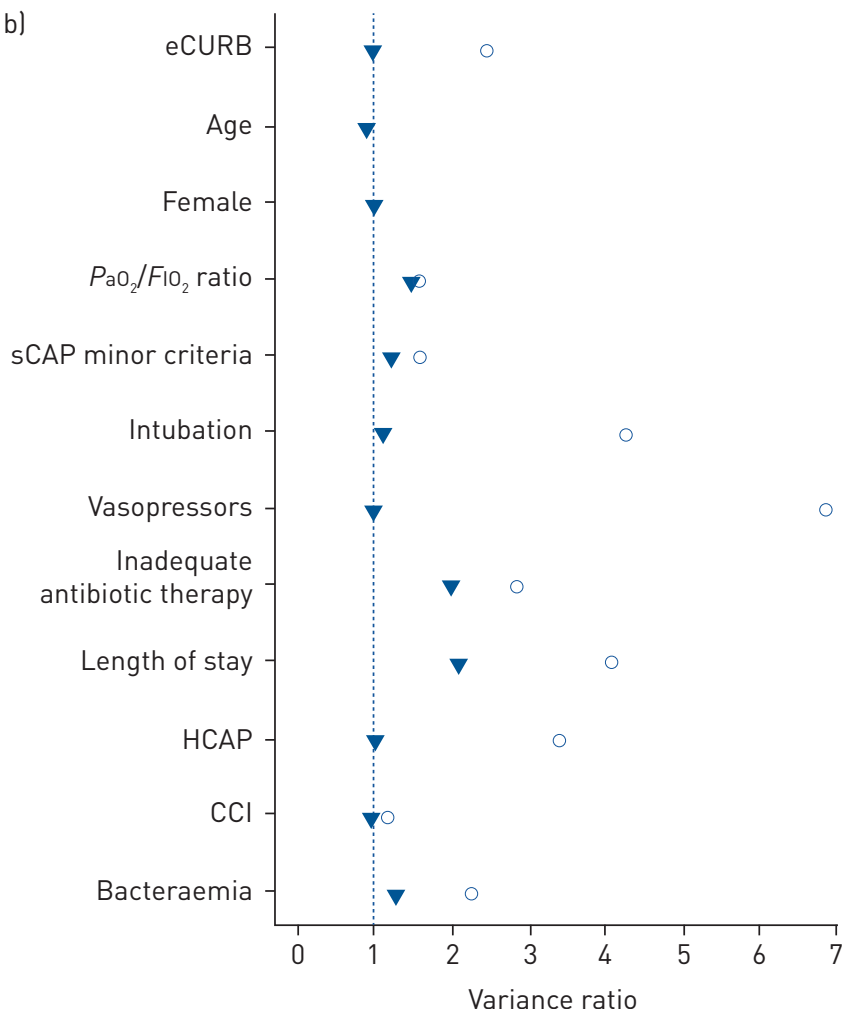

FIGURE 1 Balance diagnostics for inverse-probability of treatment weighting (IPTW). eCURB: electronic CURB-65 (confusion, urea $>7 \mathrm{mmol} \cdot \mathrm{L}^{-1}$ respiratory rate $\geqslant 30$ breaths $\mathrm{min}^{-1}$, blood pressure $<90 \mathrm{mmHg}$ (systolic) or $\leqslant 60 \mathrm{mmHg}$ (diastolic), age $\geqslant 65$ years) predicted 30 -day mortality; $\mathrm{PaO}_{2}$ : arterial oxygen tension; $\mathrm{FlO}_{2}$ : inspiratory oxygen fraction; SCAP: severe community-acquired pneumonia [12]; HCAP: healthcare-associated pneumonia; CCl: Charlson Comorbidity Index; ATT: average treatment effects on the treated. In principle, IPTW should shrink a) the standardised difference (of the first central moment) toward 0 and b) the variance ratio (of the second central moment) toward 1 . Observing both panels, we see that the IPTW tended to improve the balance between broad-spectrum antibiotic use across all covariates.

outcomes and antibiotic prescribing are influenced by provider, and our consequent choice to adjust for baseline provider variability in our primary analysis. In both the primary and the IPTW multivariable regression models, broad-spectrum antibiotic use remained associated with mortality, while HCAP status did not.

The finding that HCAP status itself contributes less to overall mortality than do severity of illness, comorbidities, antibiotic selection and goals of care aligns with the conclusion of a meta-analysis by CHALmers et al. [8]. In a large healthcare database study, RothBerg et al. [7] reported persistently increased case fatality rate in HCAP compared with CAP despite adjustment for demographic, comorbidity and severity data. However, neither of these studies included adjustment for broad-spectrum or inadequate initial antibiotic spectrum.

One strength of this study was the use of a multilevel propensity score model to account for some provider variability and confounders, as well as IPTW, which, in principle, balances treatment and non-treatment groups to theoretically simulate results from a randomised controlled trial [18]. After IPTW adjustment, the average effect on mortality and other outcomes in patients treated with broad-spectrum antibiotics not only persisted, but was amplified, suggesting that the association is robust.

This observation that empiric broad-spectrum antibiotics may be associated with worsened outcomes is intriguing and merits further investigation. Previously, ATTRIDGE et al. [10] showed that guidelineconcordant broad-spectrum antibiotics were associated with increased 30-day mortality (OR 2.1, 95\% CI 1.86-2.55) after propensity score adjustment in a US Veterans Affairs cohort of 15071 non-critically ill HCAP patients. Similarly, Rothberg et al. [9] found that broad-spectrum antibiotic use remained associated with increased odds (OR 1.39, 95\% CI 1.32-1.47) of inpatient mortality after adjustment for a large set of clinical/demographic features and propensity adjustment in 85097 patients in an administrative database. Neither study adjusted for microbiology and both studies acknowledged that their observations may have been influenced by unmeasured confounders unavailable in administrative databases. 
Antibiotics are associated with multiple consequences that could potentially impact outcomes, including hypersensitivity, gastrointestinal disturbances, encephalopathy, cytopenias, disruption of the microbiome and CDI [20-24]. Broad-spectrum regimens are also associated with increased length of stay, which could also increase cumulative exposure to other nosocomial insults that impact outcomes. Adverse drug events complicate up to $15 \%$ of hospital admissions [25] and antibiotics are among the most common culprits [23]. In pneumonia, antibiotic-associated adverse drug events are associated with longer length of stay [26]. Among adverse drug effects, acute kidney injury is most relevant to broad-spectrum agents. Recently, it has been identified that the most common broad-spectrum regimen used in pneumonia, i.e. vancomycin plus piperacillin-tazobactam, is associated with significant risk of nephrotoxicity [27-32]. A meta-analysis including nearly 25000 patients indicated that the rate of acute kidney injury with this regimen is $>21 \%$ and more than twice as likely compared with vancomycin alone or in combination with other $\beta$-lactams, with a number needed to harm of only 11 [33]. Acute kidney injury is a known contributor to mortality in pneumonia [34]. Considering that vancomycin and piperacillin-tazobactam use has doubled in the last decade $[4,35]$, this observation is important.

CDI is more likely with broad-spectrum regimens that include more than one antibiotic class [20]. Indeed, in our study, odds of CDI were four-fold greater for those receiving broad-spectrum regimens. Like acute kidney injury, CDI is also associated with increased mortality in pneumonia [36]. Similarly, impact of antibiotics on the microbiome could worsen outcomes in ways more difficult to identify. Secondary or subsequent infections are more common in patients exposed to antibiotics, especially those with the greatest activity against components of the microbiome [37]. BAGGS et al. [21] recently showed that the risk of 90-day readmission with sepsis or septic shock was $65 \%$ higher in patients who had received broad-spectrum antibiotics.

In that context, results from our manual review of mortality cases are particularly interesting. While comorbidities and complications, palliative goals of care, advanced age, and severity were the most commonly identified contributors to demise, antibiotic side-effects, including encephalopathy, acute kidney injury and CDI, were identified as having a plausible contribution to mortality in $17.5 \%$ of cases. This suggests that outcomes noted in our statistical analyses may have a real-world basis.

Prescription of broad-spectrum antibiotics far exceeds rates of DRP recovery in pneumonia. The lack of improved outcomes with widespread use of broad-spectrum regimens supports the notion that most culture-negative pneumonia is not due to occult DRPs, even in patients with epidemiological risk of resistance [5]. Clinical decision support tools to guide appropriate selection of broad-spectrum regimens in patients with risk factors for drug-resistant bacteria are important [38], as are rapid methods of excluding the presence of resistant pathogens [39]. The pendulum may now be swinging back toward more narrow empiric antibiotic prescribing for community-onset pneumonia.

This study is limited by its observational design. Despite the inclusion of a large set of well-recognised confounder variables and use of IPTW to balance treatment groups in an effort to mitigate indication bias, it is possible that the propensity model was not well specified, due perhaps to poor model assumptions or unmeasured variables. Two such variables identified in our manual review include complications of hospitalisation and palliative goals of care, which are known predictors of mortality. However, these variables could only introduce bias if they are associated with the decision to prescribe initial empiric broad-spectrum antibiotics, which seems unlikely for these variables. Another possible limitation was the inability to accurately capture "do not intubate/do not resuscitate" status. While goals of care certainly contribute to mortality, code status does not uniformly determine the level of care provided and reflects a dynamic decision that may change during the course of a hospital admission. For example, in our hospitals, patients with "do not resuscitate" status are regularly admitted for aggressive care in the ICU, while some patients with no code status on admission change to "do not intubate" or palliative status later in their course. For this reason, we conducted the ICU subgroup analysis. We were unable to accurately report the cause of mortality in all cases and, in this cohort, vasopressor use was used as a surrogate for septic shock. It is also possible that the manual chart review was prone to some bias because it was conducted by a single, non-blinded investigator. A strength of this study was the pragmatic real-world design that permitted the inclusion of severely ill patients and those with decreased level of consciousness, yielding a study population more representative of emergency department pneumonia patients than prospective cohorts that require individual consent.

\section{Conclusion}

Whether analysed by unweighted multivariable regression or by IPTW, use of broad-spectrum antibiotics in community-onset pneumonia was associated with higher mortality, longer hospital stay, higher cost and increased risk of CDI. These results lend additional support for more judicious use of broad-spectrum antibiotics in community-onset pneumonia. Accurate methods to better identify the small proportion of pneumonia patients who require broad-spectrum antibiotics are needed. 
Acknowledgements: Some of the data were presented at the European Respiratory Society International Congress, Paris, September 17, 2018.

Author contributions: B.J. Webb had full access to all the data in the study, and takes responsibility for the integrity of the data and the accuracy of the data analysis. Study concept and design: B.J. Webb and N.C. Dean. Acquisition, analysis or interpretation of data: all authors. Drafting of the manuscript: B.J. Webb, J. Sorensen and N.C. Dean. Critical review of the manuscript for important intellectual content: all authors. Statistical analysis: J. Sorensen and B.J. Webb.

Conflict of interest: B.J. Webb reports research support from Astellas Pharma, Inc. unrelated to this study and speaking honorarium from BioFire Diagnostics. J. Sorensen has nothing to disclose. A. Jephson has nothing to disclose. I. Mecham has nothing to disclose. N.C. Dean has nothing to disclose.

Support statement: A portion of this study was funded by a grant from the Intermountain Research and Medical Foundation. The Intermountain Research and Medical Foundation provided internal funding to support study coordinator salary and statistical analysis. No investigator salary support was provided. The funding body had no role in the design and conduct of the study; collection, management, analysis and interpretation of the data; preparation, review or approval of the manuscript; and decision to submit the manuscript for publication. Funding information for this article has been deposited with the Crossref Funder Registry.

\section{References}

1 McCabe C, Kirchner C, Zhang H, et al. Guideline-concordant therapy and reduced mortality and length of stay in adults with community-acquired pneumonia: playing by the rules. Arch Intern Med 2009; 169: 1525-1531.

2 American Thoracic Society, Infectious Diseases Society of America. Guidelines for the management of adults with hospital-acquired, ventilator-associated, and healthcare-associated pneumonia. Am J Respir Crit Care Med 2005; 171: 388-416.

3 Dellinger RP, Levy MM, Carlet JM, et al. Surviving Sepsis Campaign: international guidelines for management of severe sepsis and septic shock: 2008. Crit Care Med 2008; 36: 296-327.

4 Jones BE, Jones M, Huttner B, et al. Trends in antibiotic use and nosocomial pathogens in hospitalized veterans with pneumonia at 128 medical centers, 2006-2010. Clin Infect Dis 2015; 61: 1403-1410.

5 Haessler S, Lagu T, Lindenauer PK, et al. Treatment trends and outcomes in healthcare-associated pneumonia. J Hosp Med 2017; 12: 886-891.

6 Dean NC, Jones BE, Jones JP, et al. Impact of an electronic clinical decision support tool for emergency department patients with pneumonia. Ann Emerg Med 2015; 66: 511-520.

7 Rothberg MB, Haessler S, Lagu T, et al. Outcomes of patients with healthcare-associated pneumonia: worse disease or sicker patients? Infect Control Hosp Epidemiol 2014; 35: Suppl. 3, S107-S115.

8 Chalmers JD, Rother C, Salih W, et al. Healthcare-associated pneumonia does not accurately identify potentially resistant pathogens: a systematic review and meta-analysis. Clin Infect Dis 2014; 58: 330-339.

9 Rothberg MB, Zilberberg MD, Pekow PS, et al. Association of guideline-based antimicrobial therapy and outcomes in healthcare-associated pneumonia. J Antimicrob Chemother 2015; 70: 1573-1579.

10 Attridge RT, Frei CR, Restrepo MI, et al. Guideline-concordant therapy and outcomes in healthcare-associated pneumonia. Eur Respir J 2012; 38: 878-887.

11 Mecham ID DN, Wilson EL, Jephson AR, et al. The association of qSOFA, SOFA and SIRSA with mortality in emergency department pneumonia patients. J Respir Infect 2018; 97: e12634.

12 Mandell LA, Wunderink RG, Anzueto A, et al. Infectious Diseases Society of America/American Thoracic Society consensus guidelines on the management of community-acquired pneumonia in adults. Clin Infect Dis 2007; 44: Suppl. 2, S27-S72.

13 McCauley LM, Webb BJ, Sorensen J, et al. Use of tracheal aspirate culture in newly intubated patients with community-onset pneumonia. Ann Am Thorac Soc 2016; 13: 376-381.

14 Jones BE, Jones J, Bewick T, et al. CURB-65 pneumonia severity assessment adapted for electronic decision support. Chest 2011; 140: 156-163.

15 Sanz F, Dean N, Dickerson J, et al. Accuracy of $\mathrm{PaO}_{2} / \mathrm{FIO}_{2}$ calculated from $\mathrm{SpO}_{2}$ for severity assessment in ED patients with pneumonia. Respirology 2015; 20: 813-818.

16 Guo S, Fraser MG. Propensity Score Analysis: Statistical Methods and Applications (Advanced Quantitative Techniques in the Social Sciences). Thousand Oaks, Sage, 2014.

17 Mao H, Li L, Greene T. Propensity score weighting analysis and treatment effect discovery. Stat Methods Med Res 2018; in press [https://doi.org/10.1177/0962280218781171].

18 Austin PC, Stuart EA. Moving towards best practice when using inverse probability of treatment weighting (IPTW) using the propensity score to estimate causal treatment effects in observational studies. Stat Med 2015; 34: 3661-3679.

19 Stasinopoulos DM, Rigby RA. Generalized additive models for location scale and shape (GAMLSS) in R. J Stat Softw 2007; 23: 1-46.

20 Chalmers JD, Al-Khairalla M, Short PM, et al. Proposed changes to management of lower respiratory tract infections in response to the Clostridium difficile epidemic. J Antimicrob Chemother 2010; 65: 608-618.

21 Baggs J, Jernigan JA, Halpin AL, et al. Risk of subsequent sepsis within 90 days after a hospital stay by type of antibiotic exposure. Clin Infect Dis 2018; 66: 1004-1012.

22 Geller AI, Lovegrove MC, Shehab N, et al. National estimates of emergency department visits for antibiotic adverse events among adults - United States, 2011-2015. J Gen Intern Med 2018; 33: 1060-1068.

23 Shehab N, Patel PR, Srinivasan A, et al. Emergency department visits for antibiotic-associated adverse events. Clin Infect Dis 2008; 47: 735-743.

24 Vardakas KZ, Kalimeris GD, Triarides NA, et al. An update on adverse drug reactions related to beta-lactam antibiotics. Expert Opin Drug Saf 2018; 17: 499-508.

25 Hug BL, Witkowski DJ, Sox CM, et al. Adverse drug event rates in six community hospitals and the potential impact of computerized physician order entry for prevention. J Gen Intern Med 2010; 25: 31-38. 
Lin RY, Nuruzzaman F, Shah SN. Incidence and impact of adverse effects to antibiotics in hospitalized adults with pneumonia. J Hosp Med 2009; 4: E7-E15.

27 Anderson CW, Cazares KS, Lustik MB, et al. Vancomycin vs. vancomycin/piperacillin-tazobactam-associated acute kidney injury in noncritically ill patients at a tertiary care military treatment facility. Mil Med 2017; 182: e1773-e1778.

28 Gomes DM, Smotherman C, Birch A, et al. Comparison of acute kidney injury during treatment with vancomycin in combination with piperacillin-tazobactam or cefepime. Pharmacotherapy 2014; 34: 662-669.

29 Mullins BP, Kramer CJ, Bartel BJ, et al. Comparison of the nephrotoxicity of vancomycin in combination with cefepime, meropenem, or piperacillin/tazobactam: a prospective, multicenter study. Ann Pharmacother 2018; 52: 639-644

30 Rutter WC, Burgess DR, Talbert JC, et al. Acute kidney injury in patients treated with vancomycin and piperacillin-tazobactam: a retrospective cohort analysis. J Hosp Med 2017; 12: 77-82.

31 Rutter WC, Burgess DS. Incidence of acute kidney injury among patients treated with piperacillin-tazobactam or meropenem in combination with vancomycin. Antimicrob Agents Chemother 2018; 62: e00264-18.

32 Rutter WC, Cox JN, Martin CA, et al. Nephrotoxicity during vancomycin therapy in combination with piperacillin-tazobactam or cefepime. Antimicrobial Agents Chemother 2017; 61: e02089-16.

33 Luther MK, Timbrook TT, Caffrey AR, et al. Vancomycin plus piperacillin-tazobactam and acute kidney injury in adults: a systematic review and meta-analysis. Crit Care Med 2018; 46: 12-20.

34 Chawla LS, Amdur RL, Faselis C, et al. Impact of acute kidney injury in patients hospitalized with pneumonia. Crit Care Med 2017; 45: 600-606.

35 Baggs J, Fridkin SK, Pollack LA, et al. Estimating national trends in inpatient antibiotic use among US hospitals from 2006 to 2012. JAMA Intern Med 2016; 176: 1639-1648.

36 Carrabba M, Zarantonello M, Formica S, et al. Pneumonia and Clostridium difficile infection: hospital acquired infection in a non-ICU department. Eur Respir J 2012; 40: Suppl. 56, 1201-1210.

37 Malik U, Armstrong D, Ashworth M, et al. Association between prior antibiotic therapy and subsequent risk of community-acquired infections: a systematic review. J Antimicrob Chemother 2018; 73: 287-296.

38 Webb BJ, Dascomb K, Stenehjem E, et al. Derivation and multicenter validation of the drug resistance in pneumonia clinical prediction score. Antimicrob Agents Chemother 2016; 60: 2652-2663.

39 Dangerfield B, Chung A, Webb B, et al. Predictive value of methicillin-resistant Staphylococcus aureus (MRSA) nasal swab PCR assay for MRSA pneumonia. Antimicrob Agents Chemother 2014; 58: 859-864. 